\title{
Physiological effects and subjective tolerability of prone positioning in COVID-19 and healthy hypoxic challenge
}

\author{
Akhilesh Jha $\mathbb{C}^{1,2,6}$, Fangyue Chen ${ }^{2,6}$, Sam Mann ${ }^{3}$, Ravi Shah $\mathbb{D}^{2}$, Randa Abu-Youssef ${ }^{1}$, Holly Pavey ${ }^{1}$, \\ Helen Lin-Jia-Qi $\mathbb{1}^{3}$, Josh Cara ${ }^{3}$, Daniel Cunningham ${ }^{4}$, Kate Fitzpatrick ${ }^{3}$, Celine $\mathrm{Goh}^{3}$, Renee $\mathrm{Ma}^{3}$,

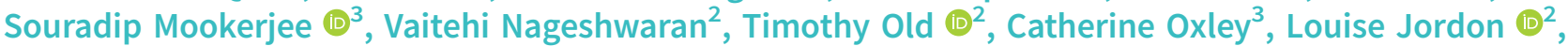 \\ Mayurun Selvan ${ }^{2}$, Anna Wood $\mathbb{D}^{3}$, Andrew Ying ${ }^{3}$, Chen Zhang ${ }^{3}$, Dariusz Wozniak ${ }^{5}$, lain Goodhart ${ }^{4}$, \\ Frances Early ${ }^{2}$, Marie Fisk ${ }^{1,2}$ and Jonathan Fuld ${ }^{2}$
}

${ }^{1}$ Dept of Medicine, University of Cambridge, Cambridge, UK. ${ }^{2}$ Dept of Respiratory Medicine, Cambridge University Hospitals NHS Foundation Trust, Cambridge, UK. ${ }^{3}$ School of Clinical Medicine, University of Cambridge, Cambridge, UK. ${ }^{4}$ Dept of Anaesthesia and Critical Care, Cambridge University Hospitals NHS Foundation Trust, Cambridge, UK. ${ }^{5}$ Respiratory Support and Sleep Centre, Royal Papworth Hospital, Cambridge, UK. ${ }^{6}$ These authors contributed equally.

Corresponding author: Akhilesh Jha (aj580@cam.ac.uk)

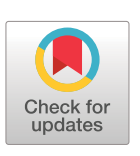

Copyright @The authors 2022

This version is distributed under the terms of the Creative Commons Attribution NonCommercial Licence 4.0. For commercial reproduction rights and permissions contact permissions@ersnet.org

Received: 25 Aug 2021 Accepted: 26 Oct 2021

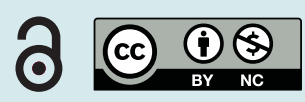

\section{Shareable abstract (@ERSpublications)}

Awake prone positioning in patients with \#COVID19 causes a small increase in $\mathrm{SpO}_{2}$ within 10 min of proning but is associated with increased subjective discomfort, and challenges in the identification of hospital patients suitable for the procedure https://bit.ly/3wg84BC

Cite this article as: Jha A, Chen F, Mann S, et al. Physiological effects and subjective tolerability of prone positioning in COVID-19 and healthy hypoxic challenge. ERJ Open Res 2022; 8: 00524-2021 [DOI: 10.1183/23120541.00524-2021].

\section{Abstract}

Background Prone positioning has a beneficial role in coronavirus disease 2019 (COVID-19) patients receiving ventilation but lacks evidence in awake non-ventilated patients, with most studies being retrospective, lacking control populations and information on subjective tolerability.

Methods We conducted a prospective, single-centre study of prone positioning in awake non-ventilated patients with COVID-19 and non-COVID-19 pneumonia. The primary outcome was change in peripheral oxygenation in prone versus supine position. Secondary outcomes assessed effects on end-tidal $\mathrm{CO}_{2}$, respiratory rate, heart rate and subjective symptoms. We also recruited healthy volunteers to undergo proning during hypoxic challenge.

Results 238 hospitalised patients with pneumonia were screened; 55 were eligible with 25 COVID-19 patients and three non-COVID-19 patients agreeing to undergo proning - the latter insufficient for further analysis. 10 healthy control volunteers underwent hypoxic challenge. Patients with COVID-19 had a median age of 64 years (interquartile range 53-75). Proning led to an increase in oxygen saturation measured by pulse oximetry $\left(\mathrm{SpO}_{2}\right)$ compared to supine position (difference $+1.62 \%$; $\mathrm{p}=0.003$ ) and occurred within $10 \mathrm{~min}$ of proning. There were no effects on end-tidal $\mathrm{CO}_{2}$, respiratory rate or heart rate. There was an increase in subjective discomfort $(p=0.003)$, with no difference in breathlessness. Among healthy controls undergoing hypoxic challenge, proning did not lead to a change in $\mathrm{SpO}_{2}$ or subjective symptom scores.

Conclusion Identification of suitable patients with COVID-19 requiring oxygen supplementation from general ward environments for awake proning is challenging. Prone positioning leads to a small increase in $\mathrm{SpO}_{2}$ within $10 \mathrm{~min}$ of proning though is associated with increased discomfort.

\section{Introduction}

The coronavirus disease 2019 (COVID-19) pandemic has caused significant global morbidity and mortality. Whilst most individuals have mild infection, a significant proportion develop hypoxia and some progress to respiratory failure. Supplemental oxygen remains the mainstay of treatment for COVID-19 patients and the delivery of adequate oxygen capacity has become a critical issue during times of peak infection [1]. There remains a need for evidence of non-pharmacological interventions, particularly in resource-poor settings. 
Prone positioning is considered standard care in patients with severe acute respiratory distress syndrome (ARDS) by increasing the arterial partial pressure of oxygen $\left(\mathrm{PaO}_{2}\right)$ /inspiratory oxygen fraction $\left(\mathrm{FiO}_{2}\right)$ (PF) ratio and reducing mortality [2]. Physiologically, proning is thought to reduce atelectasis of dorsal lung regions, improve ventilation-perfusion mismatch, and reduce compression from the abdominal cavity and the mediastinum. Mortality benefit from proning has also been linked with a decreased risk of ventilator-induced lung injury [3].

In the setting of patients with non-COVID-19-related respiratory failure receiving noninvasive ventilation, a few small retrospective case studies suggest potential beneficial effects of awake prone positioning, with improved oxygenation and a reduced rate of intubation [4-6]. In awake patients with COVID-19 pneumonia, studies have shown a beneficial effect of proning on oxygenation [7]. However, most studies are limited by their retrospective nature, include minimal time-points for physiological assessment, lack data on the tolerability of the procedure and are focused on patients receiving ventilatory support (noninvasive ventilation, continuous positive airway pressure and high-flow nasal oxygen) [8, 9]. Despite this proning has been recommended by guidelines in conscious COVID-19 patients requiring supplemental oxygen only [10].

Therefore, our study aimed firstly to determine the proportion of hypoxic patients with pneumonia eligible for proning; secondly to assess the physiological effects of proning on this milder group of awake patients with pneumonia requiring only supplemental oxygen without ventilatory support (representing the majority of hospitalised patients with COVID-19); and thirdly to assess the tolerability of proning by assessing the subjective experience of those who underwent the procedure.

Methods

Study design and participants

We conducted a prospective study to comprehensively investigate the detailed physiological effects and subjective tolerability of proning in awake, spontaneously breathing, non-ventilated hypoxaemic patients with and without COVID-19 pneumonia, who were recruited from a general hospital ward environment. We also recruited healthy volunteer controls to undergo proning during hypoxic challenge. The rationale for this latter group was to specifically test the hypothesis that parenchymal consolidation (rather than hypoxia alone) was required to observe the effects of proning on physiological parameters. Furthermore, this group acted as a control comparator group to assess the impact of proning on the subjective tolerability of the procedure.

This single-centre study was conducted at Cambridge University Hospitals NHS Foundation Trust, UK, a large acute medicine hospital in the East of England. This study was funded by support from the Addenbrooke's Charitable Trust and received favourable Ethics Committee opinion from the Health Research Authority Southwest - Cornwall \& Plymouth Research Ethics Committee (20/SW/0097) and registered with ClinicalTrials.gov (NCT04589936). All participants provided written consent.

Patients were recruited from general medical wards from September 3, 2020 to February 23, 2021. Inclusion criteria were predominantly based on the United Kingdom Intensive Care Society (ICS) guidance for prone positioning of the conscious COVID-19 patient [10], including patients with: $\mathrm{FiO}_{2} \geqslant 0.24$ requiring basic respiratory support (supplemental oxygen via face mask or nasal cannula) to achieve their clinical target oxygen saturation measured by pulse oximetry $\left(\mathrm{SpO}_{2}\right)$ (e.g. $\mathrm{SpO}_{2}$ 92-96\%); confirmed COVID-19 (by nasal swab PCR test) or non-COVID pneumonia with confirmed radiological changes; able to provide informed consent, communicate and cooperate with the procedure; could rotate and adjust position independently and had no anticipated airway issues. The exclusion criteria included those with signs of respiratory distress; haemodynamic instability; unstable spinal injury or recent abdominal surgery; pregnancy; morbid obesity; neurological issues; facial injury; gastrointestinal issues such as vomiting; and any other clinical reason which may preclude entry in the opinion of the investigator.

Inclusion criteria for healthy volunteers in the hypoxic challenge sub-study were those without respiratory illness, able to cooperate with the procedure and could rotate and adjust position independently.

Electronic health records were used to collect data on demographics, anthropometrics, baseline arterial blood gas measurement if taken on admission, white cell count (WCC) and C-reactive protein (CRP) taken at a time closest to proning, COVID-19 nasal swab PCR test status, radiographic severity score from chest radiograph [11], concomitant medications for COVID-19, comorbidities and smoking status.

Procedures

Participants were asked to proceed through a cycle of position changes: starting in the supine position for $15 \mathrm{~min}$, followed by lateral position on either side for $15 \mathrm{~min}$, then prone position aiming for at least 
30 min, ending with resupination. Prompts were provided by a member of the investigator team to ensure participants change position at the appropriate time. Proning was discontinued if participants were unable to tolerate the position, or due to any other clinical concerns.

Hypoxic challenge is a safe and tolerable procedure used to assess the suitability of adults with respiratory disease for flying at altitude [12]. We wished to assess the effects of proning in healthy volunteers without lung parenchymal disease undergoing hypoxic challenge. This involved delivery of $100 \%$ nitrogen through a $40 \%$ Venturi mask, which mixed with room air within the mask resulting in an $\mathrm{FiO}_{2}$ of 0.15 . Hypoxic challenge would be stopped if $\mathrm{SpO}_{2}$ fell below 85\% or if participants could not tolerate the procedure.

All participants (COVID-19 pneumonia, non-COVID-19 pneumonia and healthy individuals) were fitted with a Masimo monitoring device (Masimo, Irvine, CA, USA) that enabled continuous monitoring of $\mathrm{SpO}_{2}$, end-tidal $\mathrm{CO}_{2}$, heart rate and respiratory rate.

A questionnaire with a modified visual analogue scale (VAS) and free-form questions was designed to evaluate the participant's experience during the procedure [13]. After 10-15 min in each position, the participant was asked to rate their subjective breathlessness and discomfort, with each question displayed on a 0-10 VAS. Higher rates signified increased breathlessness and discomfort. VAS scores at all positions were compared with score at the initial supine position for each participant. Free-form questions included how the participant felt in each position, investigators' observation of the participant, factors that may facilitate or impede the ability to lie in the position, and changes felt during the position.

\section{Study outcomes}

The primary outcome of the study was the change in peripheral oxygenation saturation with a fixed fraction of inspired oxygen $\left(\mathrm{FiO}_{2}\right)$ in the prone position versus supine position.

The secondary outcomes included assessment of the effects of prone position versus supine or lateral position on other physiological parameters including end-tidal $\mathrm{CO}_{2}$, respiratory rate and heart rate. The subjective experience of participants undergoing prone positioning was assessed using VAS of the degree of breathlessness and discomfort in each position compared with the VAS at the initial supine position, and free text question responses were also collated.

\section{Statistical analysis}

An estimation of sample size required to determine the effect of proning was calculated using a Wilcoxon signed-rank test, estimating an effect size of change in $\mathrm{PaO}_{2}$ of $6.6 \mathrm{mmHg}$ based on published data [14]. Although $\mathrm{SpO}_{2}$ rather than $\mathrm{PaO}_{2}$ was used for this study, similar changes in outcome measures can be anticipated. A sample size of 28 for COVID-19 and non-COVID-19 pneumonia participants was deemed suitable, with a two-sided significance level of 0.05 , power of 0.8 , allowing for a $10 \%$ dropout rate. The hypoxic challenge study was not formally powered but aimed to recruit 10 volunteers as a pragmatic number of control participants.

A linear mixed effect regression was used to model the effect of position on the various outcome variables as it allows for correlation between the repeated measures across individuals. Position was included as a fixed effect and the interaction between the individual and their position was included as a random effect.

A further linear mixed effect model was produced as part of a post hoc sensitivity analysis. The $\mathrm{SpO}_{2}$ in each position was measured every $2 \mathrm{~s}$, and for each individual, summarised to a single value (median) and used as the outcome measurement. A multiple regression model was created to analyse which variables influence the magnitude of the $\mathrm{SpO}_{2}$ change associated with proning.

In order to provide insight into whether a longer duration of proning might improve $\mathrm{SpO}_{2}$, the median $\mathrm{SpO}_{2}$ in the last $60 \mathrm{~s}$ of the prone position was compared to the median $\mathrm{SpO}_{2}$ for the entire prone position using a paired two sample t-test.

The VAS data were analysed using Friedman test with Dunn’s multiple comparison.

Analysis and figures were produced using R and the following packages: tidyverse, ImerTest, and tableone, and GraphPad Prism 9.1.0 for Windows (San Diego, CA, USA). 
Results

\section{Screening}

Electronic health records of 390 patients admitted for a respiratory illness were screened (figure 1). 238 patients were hospitalised for pneumonia: 191 due to COVID-19 and 47 without COVID-19 pneumonia. 142 (74.3\%) COVID-19 patients and 41 (87.2\%) non-COVID-19 pneumonia patients had one or more exclusion criteria, with the main reason being psychiatric or cognitive issues (25.4\% of COVID-19 patients and $29.3 \%$ of non-COVID-19 patients).

\section{Excluded patients $(n=142)$}

- Psych/cognitive issues ( $n=36)$

- No investigator available to prone $(n=18)$

- No longer requiring $O_{2}(n=15)$

- Multi-comorbidities ( $n=14)$

- Morbid obesity $(n=12)$

- Admitted to ICU $(n=10)$

- Gastrointestinal issues ( $n=5)$

- Musculoskeletal issues ( $n=5)$

- End of life care ( $n=5)$

- Unstable spine/thoracic injury $(n=4)$

- Pulmonary embolus ( $n=4)$

- Neurological issues $(n=3)$

- Respiratory distress ( $n=3$ )

- Investigator discretion ( $n=2)$

- Chest pain ( $n=2)$

- Language barrier ( $n=2)$

- Pregnancy (2nd/3rd trimester) $(n=1)$

- Breast lesion $(n=1)$
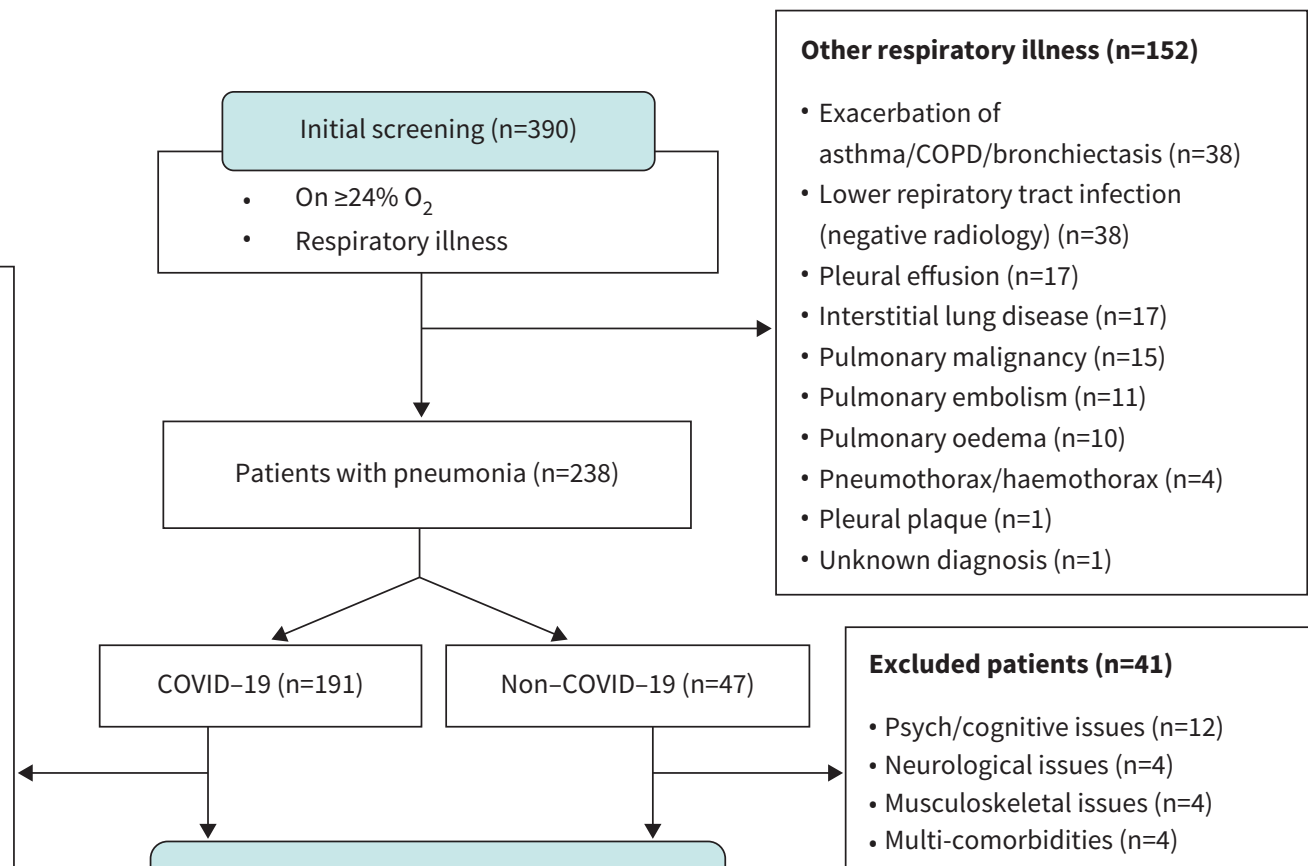

- Psych/cognitive issues ( $n=12$ )

- Neurological issues ( $n=4)$

- Musculoskeletal issues $(n=4)$

- On long-term $\mathrm{O}_{2}$ therapy $(\mathrm{n}=3)$

- Gastrointestinal issues ( $n=2)$

- No longer requiring $\mathrm{O}_{2}(\mathrm{n}=2)$

- Unstable spine/thoracic injury

- Haemodynamic instability $(n=1)$

- Acute coronary syndrome $(n=1)$

\section{Transferred to ICU ( $n=2)$}

\section{Declined ( $\mathrm{n}=\mathbf{2 2}$ )}

- Too anxious about proning $(n=5)$

- Uncomfortable with proning $(n=6)$

- Too tired or breathless $(n=4)$

- No reason given $(n=4)$

- Adverse effect proning at home $(n=1)$

- Cannot lie flat ( $n=1)$

- Unpleasant experience with clinical trials $(n=1)$
- Multi-comorbidities ( $n=4)$

- Malignancy ( $n=2)$

- End of life care $(n=2)$

- Admitted to ICU ( $n=2)$ $(n=1)$

- Chest drain in situ $(\mathrm{n}=1)$

Excluded patients $(n=41)$ Declined $(n=3)$

Unpleasant experience with clinical trials $(n=1)$

Too tired $(\mathrm{n}=1)$

New-onset diarrhoea $(n=1)$ 
49 COVID-19 patients and six non-COVID-19 pneumonia patients were approached, of which the study procedure was performed on 25 COVID-19 patients and three non-COVID-19 pneumonia patients. The main reason for COVID-19 patients declining to participate in the trial was due to anxiety about proning (22.7\%) or feeling too tired or breathless to even attempt the procedure (18.2\%). Eleven healthy volunteers were recruited to undergo hypoxic challenge and body positioning changes, of which 10 participants were analysed. Continuous monitoring data from one COVID-19 participant and one healthy volunteer were lost due to technical issues. Non-COVID pneumonia cases were not analysed due to insufficient numbers.

\section{COVID-19 cohort}

The detailed characteristics of the study population are shown in table 1. Participants with COVID-19 had a median (IQR) age of 64 (53-75) years. Eighteen (72\%) participants were male, and the median body mass index (BMI) was $28.5(25.7-31.4) \mathrm{kg} \cdot \mathrm{m}^{-2} .10$ (40\%) were ex-smokers and one (4\%) a current smoker at the time of the study. Common comorbidities included hypertension (32\%), asthma (20\%), Type II diabetes mellitus (16\%) and ischaemic heart disease (12\%). Median $\mathrm{FiO}_{2}$ at proning was $0.32(0.28$ 0.36). Baseline blood gases showed a median $\mathrm{PaO}_{2}$ of 8.00 (7.83-9.03) kPa and arterial partial pressure of carbon dioxide of $4.50(3.98-4.60) \mathrm{kPa}$. Participants had a median WCC of $7.9(6.0-11.1) \times 10^{9} \cdot \mathrm{L}^{-1}$ and median CRP of 77 (44-141) $\mathrm{mg} \cdot \mathrm{L}^{-1}$. Participants were admitted to hospital a median of 9 (3-11) days after symptom onset and underwent proning a median of 2 (1-3) days after admission. The median radiographic severity score was 5 (4-7). All patients were treated with dexamethasone, 10 (40\%) were treated with dexamethasone without additional COVID-19 therapy and 15 (60\%) trialled various additional therapeutic regimens. Regarding clinical outcomes, one patient (4\%) required intubation, one patient (4\%) required noninvasive ventilation, one patient (4\%) died, 24 patients (96\%) were discharged and median time from proning to discharge was 7 days (IQR 4-10.5 days).

\section{Effect of position on $\mathrm{SpO}_{2}$}

Patients were instructed to remain in the prone position for at least $30 \mathrm{~min}$ if possible. 16 out of 25 patients were able to prone for at least $30 \mathrm{~min}$ with a range of $10-60 \mathrm{~min}$. The median $\mathrm{SpO}_{2}$ in the prone position was significantly higher than in the initial supine position (94.74\% prone (standard error 0.38 ), 93.12\% supine (0.53), difference $+1.62 \%$; $\mathrm{p}=0.003$, figure $2 \mathrm{a}$, supplementary table $\mathrm{S} 1$ ). $\mathrm{SpO}_{2}$ in lateral and resupination positions did not differ from initial supination. A post hoc sensitivity analysis was also performed using a single median $\mathrm{SpO}_{2}$ (as opposed to using multiple raw data points) for each position in each individual (supplementary table S2), which did not differ from the results of the full mixed effect model.

Improvement in $\mathrm{SpO}_{2}$ occurred within 10 min of proning and was sustained for the duration of the proning position (figure 2c). The $\mathrm{SpO}_{2}$ at the end of the proning position (median of the last $60 \mathrm{~s}$ ) was not significantly different from the median prone $\mathrm{SpO}_{2}$ (95\% versus $95 \%$; $\mathrm{p}=0.93$ ).

The majority of participants showed an improvement in oxygenation in the prone position, which was not maintained upon resupination (supplementary figures S1 and S2). There was no change to these results when considering only those $(n=16)$ who were in the prone position for at least 30 min (supplementary table S3). There was no effect of body position on respiratory rate, end-tidal $\mathrm{CO}_{2}$ nor heart rate in COVID-19 participants or healthy volunteers (supplementary figures S3 and S4, supplementary tables S4 and S5).

\section{Healthy volunteers}

For healthy volunteers, the median age was 35 (22.5-44) years. Two (20\%) participants were male, the median BMI was 23.9 (22.8-25.9) and only one (10\%) participant was an ex-smoker. There was no significant difference in $\mathrm{SpO}_{2}$ between positions (figure $2 \mathrm{~b}$ and supplementary table $\mathrm{S} 6$ ), and $\mathrm{SpO}_{2}$ remained constant over $30 \mathrm{~min}$ in the prone position (figure $2 \mathrm{~d}$ ).

\section{Predictors of $\mathrm{SpO}_{2}$ improvement}

We produced a linear model to assess whether selected clinical variables are able to predict the magnitude of the $\mathrm{SpO}_{2}$ change from the supine to prone position. We found that a lower baseline $\mathrm{SpO}_{2}$ was predictive of a greater improvement in $\mathrm{SpO}_{2}$ with proning $(\mathrm{p}=0.003)$. Older patients had a smaller improvement with proning ( $\mathrm{p}=0.013)$. Other variables (BMI, radiographic severity score and $\mathrm{FiO}_{2}$ at time of proning) had no significant predictive effect on the size of $\mathrm{SpO}_{2}$ improvement upon proning (supplementary table S7).

\section{Tolerability and subjective experience of prone positioning}

COVID-19 patients experienced a significant increase in discomfort in both lateral (median score 3 (IQR 1.5-4.5, $\mathrm{p}=0.035)$ ) and prone (median score 4 (IQR 2.5-5.0, p=0.003)) positions, compared with initial 
TABLE 1 Baseline demographic and clinical characteristics of patients with coronavirus disease 2019 (COVID-19) and healthy volunteers

Patients with COVID-19

Subjects $\mathrm{n}$

Baseline demographics

Age years

Sex

Female

Male

BMI $\mathrm{kg} \cdot \mathrm{m}^{-2}$

Smoking history

Current smoker

Ex-smoker

Patients with COVID-19 $(n=25)$

Baseline clinical variables

$\mathrm{FiO}_{2}$ at proning

ABG

$\mathrm{pH}$

$\mathrm{PaO}_{2} \mathrm{kPa}$

$\mathrm{PaCO}_{2} \mathrm{kPa}$

Lactate $\mathrm{mmol} \cdot \mathrm{L}^{-1}$

$\mathrm{FiO}_{2}$

Inflammatory markers

WCC $\left(10^{9} \cdot \mathrm{L}^{-1}\right)$

CRP mg. $\mathrm{L}^{-1}$

COVID-19 status

SARS-CoV-2 PCR positive

Symptom onset to admission (days)

Admission to prone (days)

Radiographic severity score

COVID-19 therapeutic regimen

Dexamethasone only

Dexamethasone+Baricitinib

Dexamethasone+Dapagliflozin+Ambrisentan

Dexamethasone+EDP1815

Dexamethasone+Ravulizumab

Dexamethasone+Remdesivir

Dexamethasone+Tocilizumab

Comorbidities

Respiratory

Asthma

COPD

Interstitial lung disease

Pulmonary tuberculosis

Cardiovascular

Atrial fibrillation

Dilated cardiomyopathy

Hypercholesterolaemia

Hypertension

Ischaemic heart disease

Peripheral vascular disease

Other significant

Connective tissue disease

Chronic kidney disease

Haematological malignancy

Hypothyroidism

Osteoarthritis

Polymyalgia rheumatica

Raynaud's disease

Rheumatoid arthritis

Type II diabetes mellitus

$7.90(6.00-11.10)$
25

Healthy volunteers

10

$64.0(53.0-75.0)$

$35.0(22.5-44.0)$

$7(28.0)$

$8(80.0)$

$18(72.0)$

$2(20.0)$

$28.50(25.70-31.40)$

$23.86(22.77-25.89)$

$\begin{array}{cc}1(4.0) & 0 \\ 9(36.0) & 1(10.0)\end{array}$

$0.32(0.28-0.36)$

$\mathrm{n}=10$

$7.43(7.40-7.47)$

$8.00(7.83-9.03)$

$4.50(3.98-4.60)$

$1.70(1.50-2.10)$

$0.28(0.22-0.33)$

$n=25$

77.00 (40.00-141.00)

$\mathrm{n}=25$

$9.0(3.0-11.0)$

$2.0(1.0-3.0)$

$5(4-7)$

$10(40.0)$

$2(8.0)$

$3(12.0)$

$1(4.0)$

$2(8.0)$

$4(16.0)$

$1(4.0)$

$5(20.0)$

$2(8.0)$

$1(4.0)$

$1(4.0)$

$2(8.0)$

$1(4.0)$

$1(4.0)$

$8(32.0)$

$3(12.0)$

$1(4.0)$

$2(8.0)$

$2(8.0)$

$1(4.0)$

$2(8.0)$

$2(8.0)$

$2(8.0)$

$1(4.0)$

$1(4.0)$

$4(16.0)$ 


\begin{tabular}{|c|c|c|}
\hline & Patients with COVID-19 & Healthy volunteers \\
\hline \multicolumn{3}{|l|}{ Outcomes } \\
\hline Death & $1(4.0)$ & \\
\hline Intubation and ventilation & $1(4.0)$ & \\
\hline Noninvasive ventilation & $1(4.0)$ & \\
\hline Discharge & $24(96)$ & \\
\hline Time from proning to discharge (days) & $7(4-10.5)$ & \\
\hline \multicolumn{3}{|c|}{$\begin{array}{l}\text { Data are } n(\%) \text { or median (IQR). Radiographic severity score: semi-quantitative score indicating extent of } \\
\text { radiographic changes with a maximum score of } 8 \text {. BMI: body mass index; FiO } \mathrm{F}_{2} \text { : fractional concentration of } \\
\text { oxygen in inspired air at point of proning; ABG: arterial blood gas; PaO } \mathrm{O}_{2} \text { : arterial partial pressure of oxygen; } \\
\mathrm{PaCO}_{2} \text { : arterial partial pressure of carbon dioxide; WCC: white cell count; CRP: C-reactive protein; SARS-CoV-2: } \\
\text { severe acute respiratory syndrome coronavirus 2; NA: not applicable. ": EDP1815 is an investigational immune } \\
\text { modulator used as part of the TACTIC-E Trial: ClinicalTrials.gov Identifier: NCTO4393246. }\end{array}$} \\
\hline
\end{tabular}

supine position (median score 2 (IQR 1-3)) (figure 3a and c). There was no significant difference in the degree of reported breathlessness in any position. Healthy volunteers undergoing hypoxic challenge did not experience a significant increase in subjective breathlessness or discomfort (figure $3 \mathrm{~b}$ and d).

Qualitative data on participant and investigator observations of proning and the factors that helped or hindered tolerability are shown in supplementary table S8. 15 out of 25 participants reported some discomfort including discomfort in arms, neck and shoulders and becoming hot. For some, it became more comfortable over time as they settled into it. Eight out of 25 participants found the position comfortable. Some found the use of additional support such as pillows helpful.

\section{Discussion}

In this prospective, single-centre study of 25 awake non-ventilated COVID-19 patients on supplementary oxygen recruited from general medical wards, we found that a brief period of proning resulted in a small but significant increase in oxygenation of $1.6 \%$ compared to supine position. However, this was associated with worse subjective tolerability with increased discomfort in this position. We did not find any significant effect of proning on end-tidal $\mathrm{CO}_{2}$, respiratory rate, heart rate or a subjective sensation of breathlessness.

Our population included patients with a median age of 64 years, a male prevalence of $72 \%$ and common comorbidities including hypertension and diabetes. This is broadly in line with the demographic features common to patients presenting with COVID-19 in the UK [15]. Therefore, although this was a single-centre study, the findings are likely to have reasonable generalisability across medical ward environments in UK hospitals. An important observation, however, is that about three-quarters of COVID-19 patients screened requiring oxygenation met the study's exclusion criteria, and in a significant proportion (25\%), this was due to the presence of cognitive or psychiatric issues (such as dementia), which would have made it difficult to reliably perform the intervention. Furthermore, of 49 suitable patients who were approached, 22 declined due to pre-existing anxiety or experience of discomfort with proning or were too tired to attempt the procedure. Of the 25 patients who were suitable to undertake proning, only 64\% were able to do so for a duration of at least $30 \mathrm{~min}$. These findings therefore highlight the significant challenges of identifying and performing proning in unwell and often older-age patients with COVID-19 in general medical ward environments, despite formal guidelines that recommend widespread use of the procedure.

Virtually all studies of proning in COVID-19 have relied on very few time-points for collection of physiological data. In contrast, our study included the use of continuous physiological monitoring of multiple parameters simultaneously, permitting greater insights into the exact physiological changes during and after proning. For example, by collecting $>50000$ data points for $\mathrm{SpO}_{2}$, we demonstrated that increases in oxygen saturation typically occur within the first $10 \mathrm{~min}$ of proning. Therefore, this may be a useful clinical guide to help identify those patients who are most likely to benefit from more extended durations of proning. Indeed, at the start of the COVID-19 pandemic, rapidly delivered pragmatic guidance on the use of proning in awake patients was published [10]. The recommendation was to trial 15 min of proning and to only continue if the patient was physiologically stable and found the procedure tolerable. The data from our study support this trial duration to assess suitability of the procedure for patients. 

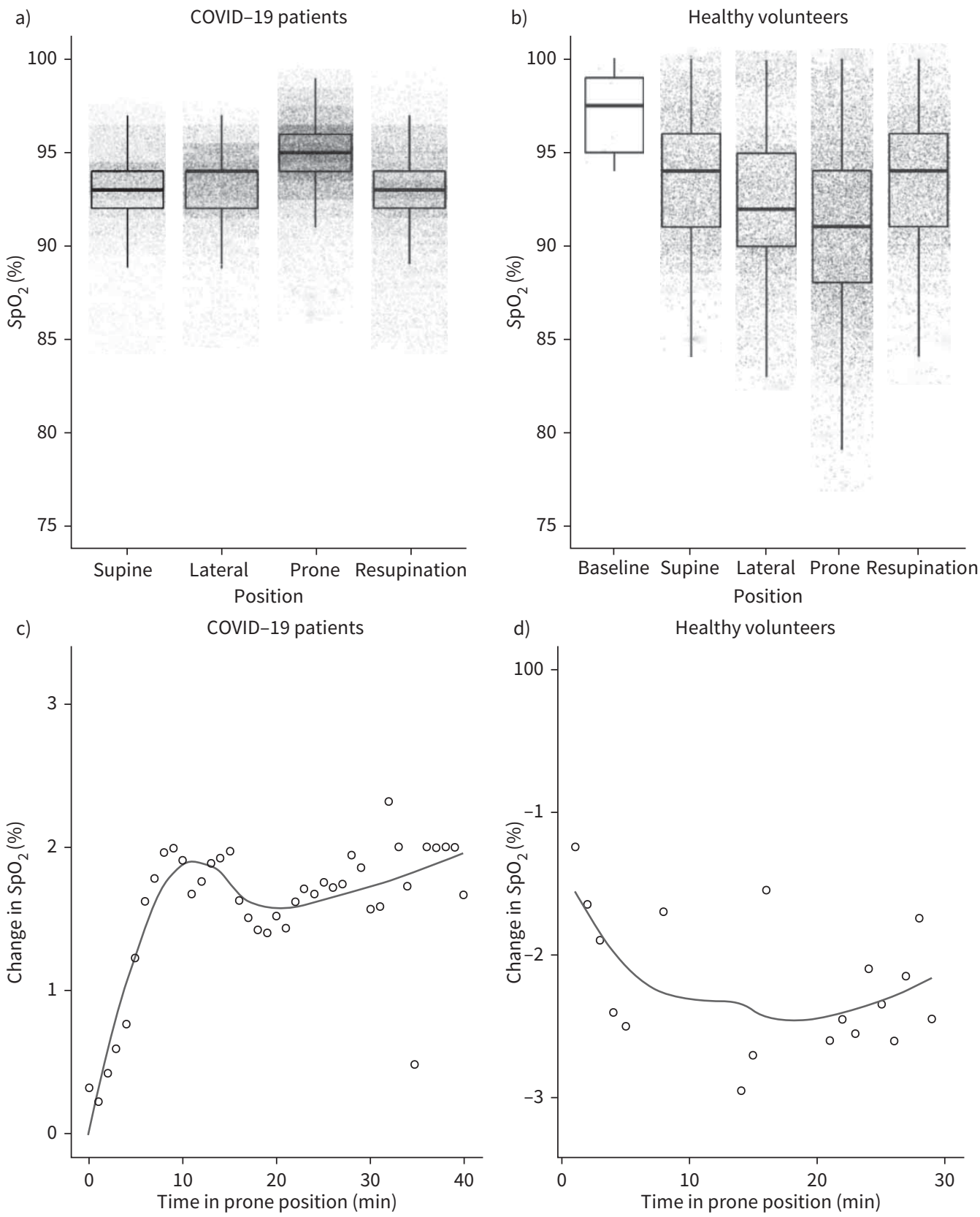

FIGURE 2 Change in oxygen saturation measured by pulse oximetry $\left(\mathrm{SpO}_{2}\right)$ in different body positions in coronavirus disease 2019 (COVID-19) pneumonia and healthy hypoxic challenge. Boxplots showing $\mathrm{SpO}_{2}$ measured in a) COVID-19 patients ( $n=24$, one dataset unavailable due to technical loss) and b) healthy volunteers $(n=10)$ in different body positions. Boxes represent 25th percentile, median and 75 th percentile. The lower and upper whiskers extend to 1.5 times the interquartile range from the 25th and 75th percentile, respectively. The overlaid dot plots show each $\mathrm{SpO}_{2}$ value that was measured (every $2 \mathrm{~s}$ ). Dot plot and LOESS smooth curve showing the absolute change in $\mathrm{SpO}_{2}$ (percentage points) over time in prone position for c) COVID-19 patients and d) healthy volunteers. The $\mathrm{SpO}_{2}$ at each timepoint in prone position was corrected for every individual's baseline $\mathrm{SpO}_{2}$ (in supine position) - each dot represents the mean corrected $\mathrm{SpO}_{2}$ in all individuals for every minute in prone position.

We collated detailed subjective symptomatic data on the tolerability of the procedure in different positions, finding that the lateral and prone positions were associated with greater discomfort, even after exclusion of a large proportion of patients due to tolerability related factors. The qualitative data suggested variability in patients' experience of proning, with the majority of people finding it uncomfortable, experiencing 
a)

COVID-19

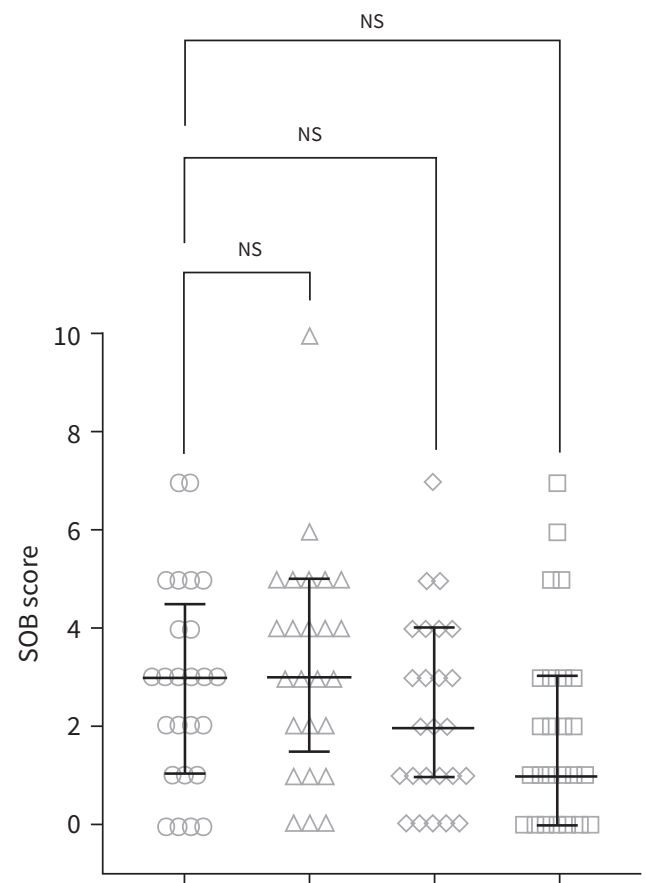

c)

NS

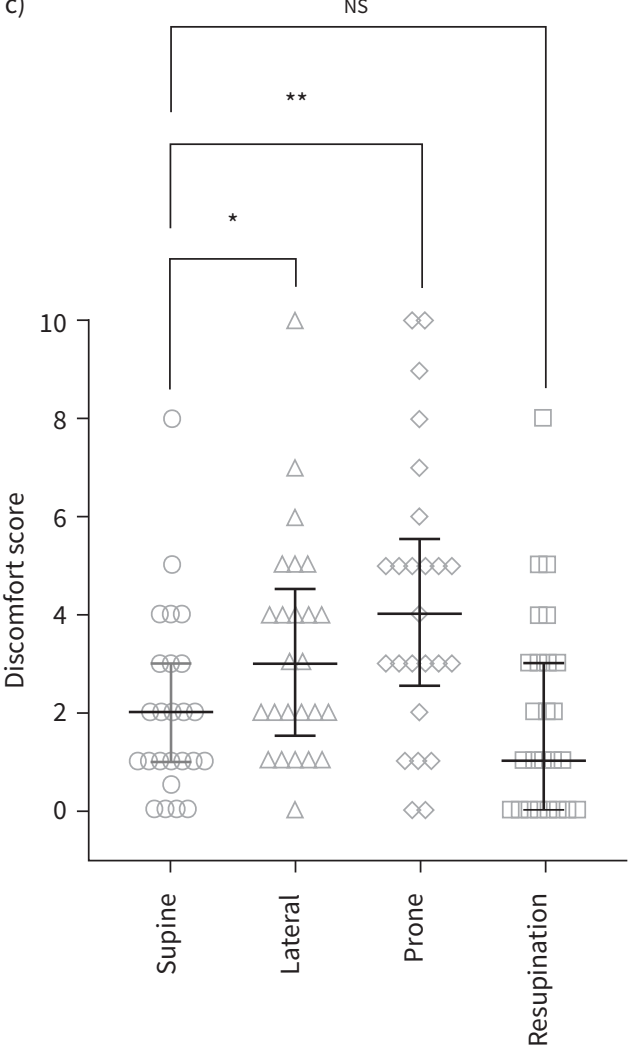

b)

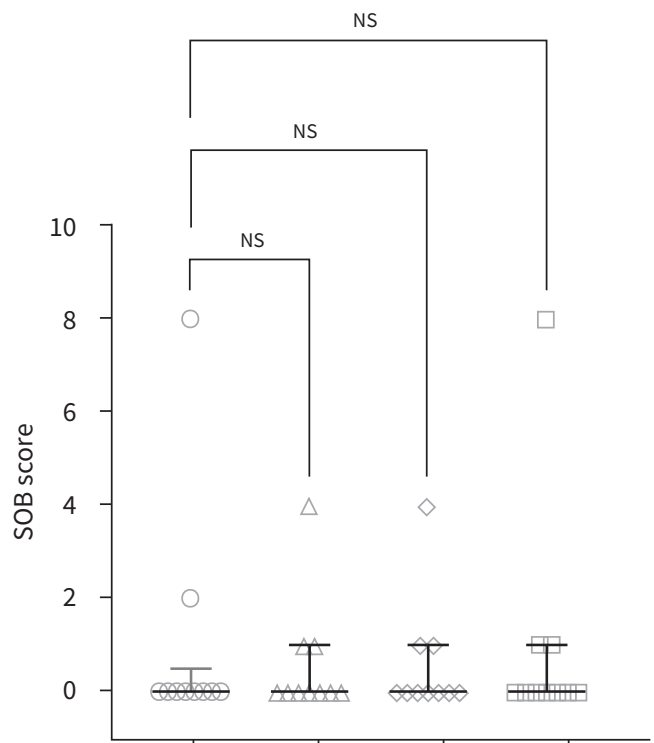

d)

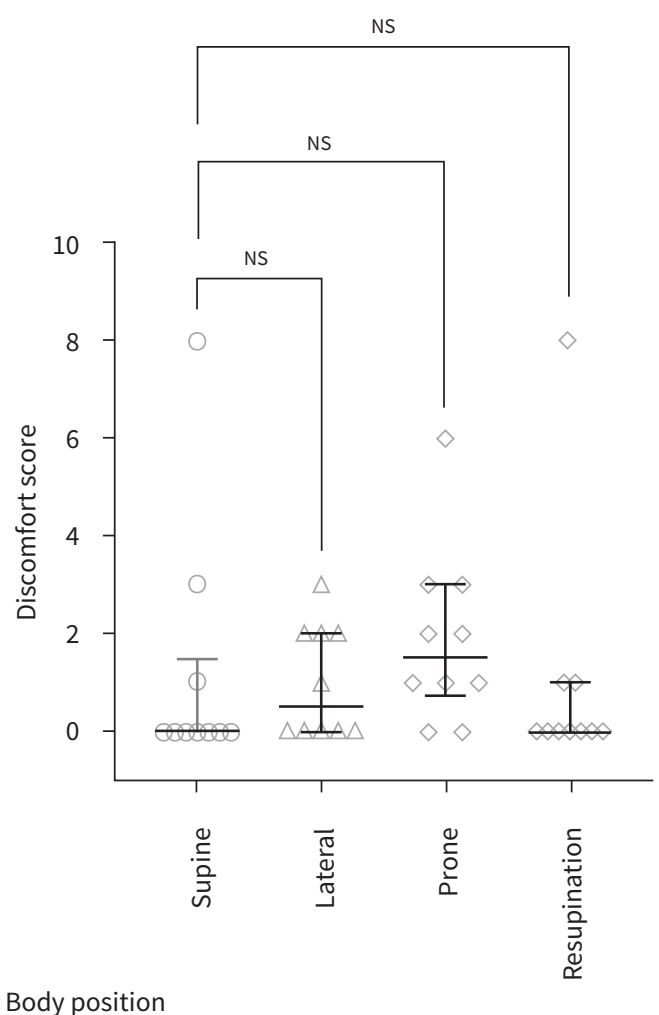

FIGURE 3 Visual analogue scales (VAS) for subjective breathlessness and discomfort in coronavirus disease 2019 (COVID-19) patients and healthy volunteers. Breathlessness (SOB) score for a) COVID-19 patients ( $n=25$ ) and b) healthy volunteers ( $n=10)$. Discomfort score for $c$ ) COVID-19 patients and d) healthy volunteers. Lines represent median (IQR). Compared using Friedman's test and Dunn's multiple correction. ${ }^{*}: p<0.05 ;{ }^{* \star}: p<0.005$. 
discomfort in the arms, neck and shoulders, as well as feeling hot. In certain individuals, breathing did become easier with increased time in the prone position, and they may benefit from a longer duration of proning. The implications for practice to help make patients more comfortable include the use of pillows for the head, neck and limbs, raising the arms forward in bed, ensuring no devices or wires are in the way and to try adjusting the angle of the bed.

Physiologically, prone positioning is thought to reduce dorsal atelectasis and compression on the lungs from the abdominal cavity, and improve ventilation-perfusion mismatch [16]. Therefore, the benefits of awake proning in respiratory failure may extend to aetiologies beyond COVID-19. In this study, we attempted to investigate the effect of awake proning on non-COVID-19 pneumonia patients. However, cases of COVID-19 pneumonia far exceeded non-COVID-19 pneumonia cases at our hospital, and we were unable to recruit sufficient numbers of non-COVID-19 patients. This was consistent with a decline in the prevalence of and mortality due to influenza and non-COVID-19 pneumonia across the UK in recent months, which for example was half the 5-year average in the month of December 2020 [17]. Nonetheless, we recruited a healthy control population with no pre-existing respiratory symptoms or clinical evidence of lung parenchymal disease and subjected them to hypoxic challenge, to mimic the conditions seen with pneumonia. Whilst the relatively small sample size precludes definite conclusions, the lack of improvement in $\mathrm{SpO}_{2}$ after proning suggests that parenchymal consolidation is required to see the full benefits of proning.

We focused on comprehensively understanding the physiological and subjective effects of a relatively brief $(<1 \mathrm{~h})$ period of proning and as such do not have data on longer durations of proning. Owing to the shorter duration of proning and the lack of randomisation, it was not appropriate to determine the impact of proning on clinical outcomes such as intensive care unit admission; randomised trials to assess this are ongoing (NCT04402879; NCT04383613).

A further limitation of the study was that it was a single-centre study, and although we used ICS guidance criteria for inclusion of appropriate patients, enrolment of centres with younger age groups and from less-well-resourced settings would provide greater generalisability.

In summary, we provide evidence that prone positioning of hospital ward-based non-ventilated awake COVID-19 patients requiring supplemental oxygenation leads to a small but significant increase in $\mathrm{SpO}_{2}$, with an improvement seen within $10 \mathrm{~min}$ of proning. Careful selection of appropriate patients and monitoring of subjective symptoms as well as physiological parameters at the early stages of the procedure are required to optimise the identification of patients who are most likely to benefit.

Acknowledgements: We would like to express gratitude to the patients who kindly took part in the study. We thank Heather Kincaid of Masimo (Irvine, CA, USA) for technical support. We are grateful to Jacqui Galloway and Tom Dymond (Cambridge University Hospitals NHS Foundation Trust, Cambridge, UK) for support with trial administration.

Provenance: Submitted article, peer reviewed.

This study is registered at www.clinicaltrials.gov with identifier number NCT04589936. We are happy to share on request the individual participant data that underlie the results reported in this article, after deidentification immediately following publication with researchers who provide a methodologically sound proposal. The study protocol and statistical analysis plan are also available.

Author contributions: Conception and design of work: A. Jha, H. Lin-Jia-Qi, D. Wozniak, I. Goodhart, F. Early, M. Fisk and J. Fuld. Acquisition of data: A. Jha, F. Chen, S. Mann, R. Shah, R. Abu-Youssef, H. Pavey, H. Lin-Jia-Qi, J. Cara, D. Cunningham, K. Fitzpatrick, C. Goh, R. Ma, S. Mookerjee, V. Nageshwaran, T. Old, C. Oxley, L. Jordon, M. Selvan, A. Wood, A. Ying, C. Zhang and M. Fisk. Analysis or interpretation of data: A. Jha, F. Chen, S. Mann, R. Shah, R. Abu-Youssef, H. Pavey, H. Lin-Jia-Qi, F. Early, M. Fisk and J. Fuld. Drafting and/or revising the article: A. Jha, F. Chen, S. Mann, R. Shah, R. Abua-Youssef, H. Pavey, D. Wozniak, I. Goodhart, F. Early, M. Fisk and J. Fuld. All authors have reviewed the manuscript and approved it for submission.

Conflict of interest: None declared.

Support statement: We are grateful for the Addenbrooke's Charitable Trust for funding this study. Funding information for this article has been deposited with the Crossref Funder Registry. 
References

1 Wiersinga WJ, Rhodes A, Cheng AC, et al. Pathophysiology, transmission, diagnosis, and treatment of Coronavirus Disease 2019 (COVID-19): a review. JAMA 2020; 324: 782-793.

2 Guérin C, Reignier J, Richard J-C, et al. Prone positioning in severe acute respiratory distress syndrome. N Engl J Med 2013; 368: 2159-2168.

3 Guérin C. Prone ventilation in acute respiratory distress syndrome. Eur Respir Rev 2014; 23: 249-257.

4 Valter C, Christensen AM, Tollund C, et al. Response to the prone position in spontaneously breathing patients with hypoxemic respiratory failure. Acta Anaesthesiol Scand 2003; 47: 416-418.

5 Feltracco P, Serra E, Barbieri S, et al. Noninvasive high-frequency percussive ventilation in the prone position after lung transplantation. Transplant Proc 2012; 44: 2016-2021.

6 Scaravilli V, Grasselli G, Castagna L, et al. Prone positioning improves oxygenation in spontaneously breathing nonintubated patients with hypoxemic acute respiratory failure: a retrospective study. $J$ Crit Care 2015; 30 : 1390-1394.

7 Prud'homme E, Trigui $\mathrm{Y}$, Elharrar $\mathrm{X}$, et al. Effect of prone positioning on the respiratory support of nonintubated patients with Coronavirus Disease 2019 and acute hypoxemic respiratory failure. Chest 2021; 160: 85-88.

8 Solverson K, Weatherald J, Parhar KKS. Tolerability and safety of awake prone positioning COVID-19 patients with severe hypoxemic respiratory failure. Can J Anaesth 2021; 68: 64-70.

9 Johnson SA, Horton DJ, Fuller MJ, et al. Patient-directed prone positioning in awake patients with COVID-19 requiring hospitalization (PAPR). Ann Am Thoracic Soc 2021; 18(8):1424-1426.

10 Bamford P, Bentley A, Dean J, et al. Intensive Care Society Guidance for Prone Positioning of the Conscious COVID Patient 2020. London, Intensive Care Society.

11 Galloway JB, Norton S, Barker RD, et al. A clinical risk score to identify patients with COVID-19 at high risk of critical care admission or death: an observational cohort study. J Infection 2020; 81: 282-288.

12 Mestry N, Thirumaran M, Tuggey JM, et al. Hypoxic challenge flight assessments in patients with severe chest wall deformity or neuromuscular disease at risk for nocturnal hypoventilation. Thorax 2009; 64: 532-534.

13 Ambrosino N, Scano G. Dyspnoea and its measurement. Breathe 2004; 1: 100-107.

14 Elharrar X, Trigui Y, Dols AM, et al. Use of prone positioning in nonintubated patients with COVID-19 and hypoxemic acute respiratory failure. JAMA 2020; 323: 2336-2338.

15 Docherty AB, Harrison EM, Green CA, et al. Features of 20133 UK patients in hospital with covid-19 using the ISARIC WHO Clinical Characterisation Protocol: Prospective observational cohort study. BMJ 2020; 369: m1985.

16 Koeckerling D, Barker J, Mudalige NL, et al. Awake prone positioning in COVID-19. Thorax 2020; 75: 833-834.

17 Office for National Statistics. Monthly mortality analysis, England and Wales: December 2020. www.ons.gov. uk/peoplepopulationandcommunity/birthsdeathsandmarriages/deaths/bulletins/monthlymortalityana lysisenglandandwales/december2020 Date last accessed: 24 May 2021. 